\title{
Desempeño y estrategia de pastoreo de ovinos pelibuey alimentados con diferentes niveles de proteína
}

\section{Performance and grazing strategy of pelibuey sheep fed with different levels of protein}

\begin{abstract}
Aldenamar Cruz-Hernández 1 (D)
Jesús Cámara-Acosta ${ }^{1}$,

Alfonso Juventino Chay-Canúl 1 (D)

Adelaido Rafael Rojas-García 2 (D)

Joel Ventura-Rios 3

Santiago Ramirez-Vera ${ }^{1}$ D

${ }^{1}$ División Académica de Ciencias

Agropecuarias. Universidad Juárez

Autónoma de Tabasco. Carretera

Villahermosa - Teapa, km $25 \mathrm{R} / \mathrm{A}$

La Huasteca Segunda Sección,

CP. 86280. Villahermosa, Tabasco,

México.

${ }^{2}$ Facultad de Ciencias Agropecuarias y Ambientales. Universidad Autónoma de Guerrero. Carretera Iguala - Tuxpan, km 2.5. Iguala de Independencia, CP. 40040. Guerrero, México.

${ }^{3}$ Universidad Autónoma Agraria Antonio Narro. Calzada Antonio Narro, CP. 25315. Buenavista, Saltillo Coahuila México.
\end{abstract}

*Autor de correspondencia: sarave2@hotmail.com

Artículo científico

Recibido: 03 de marzo 2021

Aceptado: 11 de octubre 2021

Como citar: Cruz-Hernández A Cámara-Acosta J, Chay-Canúl AJ, Rojas-García AR, Ventura-Rios J, Ramirez-Vera S (2021) Desempeño y estrategia de pastoreo de ovinos pelibuey alimentados con diferentes niveles de proteína. Ecosistemas y Recursos Agropecuarios Núm. Esp. II: e2944. DOI: 10.19136/era.a8nll.2944
RESUMEN. Con el objetivo de evaluar el desempeño de corderas Pelibuey alimentadas con diferentes niveles de proteína y pasto Tanzania a dos régimen de pastoreo rotacional. Se utilizó un diseño completamente al azar. Se utilizaron 30 corderas de la raza Pelibuey con peso vivo inicial de $20.33 \pm 1.56 \mathrm{~kg}$, que se distribuyeron en 30 corrales de $2.5 \mathrm{~m}$ de largo $\times 0.80 \mathrm{~m}$ de ancho y en una pradera de 1.5 ha divididas en 0.25 ha. Los animales se alimentaron a las 07:00 am y a las 16:00 pm. Con dietas que contenían 10,12, 14, 16\% PC y un alimento testigo, las corderas se agruparon y pastorearon en praderas a dos intensidades de $9-11 \mathrm{~cm}$ y 13- $15 \mathrm{~cm}$ de altura residual. Se avaluaron materia seca (MS), tasa de crecimiento (TC), análisis bromatológico del pasto y ganancia de peso. La mayor acumulación de MS y TC se obtuvieron en los meses de junio y julio. La altura residual de 9-11 $\mathrm{cm}$ presentó el mayor contenido de proteína cruda (PC) y fibra detergente neutro (FDN). Los valores mayores en la ganancia de peso se obtuvieron en corderas que pastaron en praderas a una altura residual de $9-13 \mathrm{~cm}$ y que se suplementaron con alimento con 14 y $16 \%$ de PC. El pasto Tanzania presentó buena producción de MS y componentes morfológicos a una intensidad de $13-15 \mathrm{~cm}$. En tanto que las corderas que se alimentaron con $16 \%$ PC en la ración presentaron las mayores ganancia diaria de peso.

Palabras clave: Panicum Maximum cv. Tanzania, intensidad de pastoreo, altura residual, régimen de pastoreo.

ABSTRACT. With the objective of evaluating the performance of Pelibuey lambs fed with different levels of protein and Tanzania grass at two rotational grazing regimes. It was used completely random design. Were used 30 ewes of the Pelibuey breed with an initial live weight of $20.33 \pm 1.56 \mathrm{~kg}$, which were distributed in 30 pens of $2.5 \mathrm{~m}$ long $\times 0.80 \mathrm{~m}$ wide and in a 1.5 ha pasture divided into 0.25 ha. The animals were fed at 07:00 am and at 16:00 pm. With diets containing 10, 12, 14, $16 \%$ CP and a control feed, the lambs were grouped and grazed in pastures at two intensities of $9-11 \mathrm{~cm}$ and $13-15 \mathrm{~cm}$ of residual height. Were evaluated dry matter (DM), growth rate (TC), bromatological analysis of the pasture and weight gain. The highest accumulation of MS and TC were obtained in the months of June and July. The residual height of $9-11 \mathrm{~cm}$ presented the highest content of crude protein (CP) and neutral detergent fiber (NDF). The highest values in weight gain were obtained in lambs that grazed in pastures at a residual height of $9-13 \mathrm{~cm}$ and that were supplemented with food with 14 and $16 \%$ CP. The Tanzania grass presented good DM production and morphological components at an intensity of $13-15 \mathrm{~cm}$. While the lambs that were fed $16 \% \mathrm{CP}$ in the ration presented the highest daily weight gains.

Key words: Panicum Maximum cv. Tanzania, grazing intensity, residual height, grazing regime. 


\section{INTRODUCCIÓN}

La producción ovina constituye una de las fuentes de alimento para satisfacer las demandas calóricas y proteicas del hombre. En México, el inventario de ovinos es de 8.6 millones de cabezas con una producción de carne de 153507 t, de las cuales el $25 \%$ corresponde a la región tropical de México (SIAP 2014). Para las regiones tropicales las razas que predominan son: Katahdin, Dorper, Blackbelly y Pelibuey (Macías et al. 2010, Hernández et al. 2009). La raza Pelibuey es una raza de pelo, de origen africano (Ulloa et al. 2009), de Cuba ingresaron a México por la Península de Yucatán entre 1930 y 1940 . Por su capacidad de adaptación se encuentran diseminada por todo México, debido a su rusticidad, docilidad, fácil manejo y exige poca inversión para mantenimiento (Avendaño et al. 2004).

En el trópico mexicano la producción de corderos en pie provienen de sistemas extensivos donde la principal fuente de alimento es el forraje, de tal manera que la producción ovina es condicionada por la disponibilidad y calidad de la misma (Nantes et al. 2013). En este sentido el manejo de las especies forrajeras tropicales debe ser considerado estratégico, para aumentar la eficiencia productiva y económica, sin deteriorar la pradera (Zanine et al. 2013). Al respecto, Hernández et al. (2002) mencionan que la frecuencia e intensidad de cosecha son los dos componentes más importantes que determinan la producción del forraje y la persistencia de la misma, la cual puede ser modificado por la carga animal (Difante et al. 2010, Inyang et al. 2010).

Las especies de pastos nativos se caracterizan por su bajo rendimiento de materia seca (MS) y valor nutritivo, en tanto que los pastos introducidos como Brachiarias ssp y Panicum ssp. tienen alta producción de MS y mejor calidad nutritiva. Mientras que el Panicum Maximum cv Tanzania es un forraje nativo de África tropical y subtropical de aspecto perenne amacollado de rizomas cortos con tallos que pueden alcanzar los $2 \mathrm{~m}$ de altura. El cual se puede encontrar hasta los 2000 sobre el nivel del mar, es resiste temporadas de secas (no mayor a 4 meses), y se adapta a terrenos bien drenados, ya que no tolera las inundaciones prolongadas (Améndola et al. 2005). Pero para los pastos introducidos las recomendaciones para utilizarlos en sistemas de producción de ovinos son casi nulas. Por lo anterior, es importante conocer el momento oportuno que la pradera debe ser cosechada, además para hacer más eficiente el sistema y atender la demanda creciente de carne ovina por los consumidores.

Se sabe que proporcionar raciones proteicas en la dieta, no solo permite al animal cubrir sus requerimientos, sino que también aumenta el desempeño animal, ya que la concentración y calidad de la proteína puede afectar el consumo. Al respecto concentraciones debajo del $12 \%$ pueden reducir la digestión de la fibra y restringir la ingesta del alimento, pero lo contrario ocurre cuando se incrementa el nivel de proteína en la dieta (Moreno et al. 2011). Por lo anterior, el objetivo del presente estudio fue evaluar el desempeño de corderas Pelibuey alimentadas con diferentes niveles de proteína y pasto Tanzania (Panicum Maximum) a dos régimen de pastoreo rotacional.

\section{MATERIALES Y MÉTODOS}

\section{Ubicación del área de estudio}

El experimento se desarrolló en el Rancho el Rodeo, ubicado en la ranchería Víctor Manuel Fernández Manero, primera sección del municipio de Jalapa, Tabasco, se localiza en las coordenadas geográficas a $17^{\circ} 46^{\prime} 56^{\prime \prime}$ LN y $92^{\circ} 57^{\prime} 28^{\prime \prime}$ LO, a una altura de 19.8 metros sobre nivel del mar, con suelo Luvisol crómico (Palma y Cisneros 1996). De acuerdo al sistema Koeppen modificado por García (2004), el clima es cálido húmedo $\operatorname{Af}(\mathrm{m}) \mathrm{w}(\mathrm{i})$, con altas precipitaciones en el verano. El periodo de evaluación comprendió de febrero a junio del 2014.

\section{Manejo de los animales}

Se utilizó una galera con una superficie de 100 $\mathrm{m}^{2}$ (20 m x $5 \mathrm{~m}$ ) que fue dividida en 30 corrales de $2.5 \mathrm{~m}$ de largo $\times 0.80 \mathrm{~m}$ de ancho, con bebedero y comedero. También se delimitó una pradera de 1.5 ha de pasto Panicum Maximum cv. Tanzania que se dividió en superficies de 0.25 ha. Además de 
30 corderas de la raza Pelibuey con peso vivo inicial de $20.33 \pm 1.56 \mathrm{~kg}$, que se desparasitaron con Clorhidrato de Levamisol al $12 \%$, y se les administraron vitaminas $A D E$, posteriormente las borregas se identificadas con aretes y collares. Como alimento se les proporcionaron $300 \mathrm{~g}$ de alimento a cada cordera antes y después del pastoreo como adaptación a la dieta durante un periodo de 15 días, después se incrementó la asignación de alimento de acuerdo al consumo.

Las dietas se ofrecieron para su consumo durante $2 \mathrm{~h}$, dos veces al día a las 07:00 am y 16:00 pm (Tabla 1). Todas las corderas después de ofrecerles el alimento por la mañana salían a pastorear, de acuerdo al tratamiento correspondiente donde se le daba agua a libre acceso y por las tardes se encerraban en corrales individuales.

\section{Manejo de las praderas}

Se utilizó una superficie de $15000 \mathrm{~m}^{2}$ que se dividió en dos sub-parcelas, cada sub-parcela se dividió en tres cuarteles de $2500 \mathrm{~m}^{2}$. Los tratamientos fueron dos intensidades de pastoreo (IP) de $9-11 \mathrm{~cm}$ y $13-15 \mathrm{~cm}$ de altura y a una frecuencia de pastoreo (FP) de 21 días, combinados con dietas de 10, 12, 14 y $16 \%$ PC en la ración y un alimento testigo (comercial).

Los animales se distribuyeron en 10 grupos experimentales de acuerdo al nivel de proteína y al manejo de potrero asignado; correspondiendo 3 animales para cada tratamiento con tres repeticiones. $\mathrm{T} 1$ = Alimento testigo + IP de 9-11 cm de altura, T2 $=$ Alimento testigo $+\mathrm{IP}$ de $13-15 \mathrm{~cm}$ de altura, $\mathrm{T} 3=$ Alimento con $10 \%$ de PC + IP de $9-11 \mathrm{~cm}$ de altura residual, T4 = Alimento con 10\% de PC + IP de 13-15 $\mathrm{cm}$ de altura, $\mathrm{T} 5=$ Alimento con $12 \%$ de PC + IP de 9-11 $\mathrm{cm}$ de altura, T6 = Alimento con $12 \%$ de PC + IP de $13-15 \mathrm{~cm}$ de altura, T7 = Alimento con $14 \%$ de $\mathrm{PC}+\mathrm{IP}$ de $9-11 \mathrm{~cm}$ de altura, T8 = Alimento con $14 \%$ de PC + IP de 13-15 cm de altura, T9 = Alimento con $16 \%$ de $\mathrm{PC}+\mathrm{IP}$ de $9-11 \mathrm{~cm}$ de altura, T10 = Alimento con $16 \%$ de PC + IP de $13-15 \mathrm{~cm}$ de altura.

\section{Variables evaluadas \\ Acumulación de materia seca (MS)}

En cada cuartel se colocaron dos cuadrantes fijos de $0.25 \mathrm{~m}^{2}(50 \times 100 \mathrm{~cm})$ que permanecieron durante el experimento. Un día antes de que los ovinos pastorearan las praderas, se cosechaban los dos cuadrantes a la altura residual correspondiente, las muestras colectadas se pesaron en una balanza digital, de cada muestra se tomaron una sub-muestra de $200 \mathrm{~g}$ de peso en verde, que se separaron manualmente en hoja (lámina foliar), tallo (tallo + vaina foliar) y material muerto. Después de separar los componentes morfológicos se pesaron en una balanza digital Scout Pro, y se metieron en estufas de aire forzado a $65^{\circ} \mathrm{C}$ hasta peso constante todas las muestras y submuestras. Los valores de masa de forraje, se convirtieron en $\mathrm{kg} \mathrm{MS} \mathrm{ha}^{-1}$ y los componentes morfológicos se expresarán en porcentaje (\%) de la masa de forraje (Cruz-Sánchez et al. 2018).

\section{Tasa de crecimiento de forraje}

Para calcular la tasa de crecimiento se empleó los datos de forraje cosechado antes de cada pastoreo, utilizando la siguiente fórmula: $\mathrm{TCF}=\mathrm{FC} / \mathrm{t}$. Donde, TCF = tasa de acumulación de forraje (kg MS ha $^{-1}$ día $\left.^{-1}\right)$; FC=forraje cosechado $\left(\mathrm{kg} \mathrm{MS} \mathrm{ha}^{-1}\right) ; \mathrm{t}=$ días transcurridos entre pastoreos (Cruz-Sánchez et al. 2018).

\section{Calidad del forraje y respuesta productiva}

Proteína, se determinó mediante el método de Microkjendhal (AOAC 2005) y el valor de Nitrógeno, se multiplicó por 6.25, para obtener el contenido de proteína total. Fibra detergente neutro y fibra detergente ácido, se obtuvo, de acuerdo a la metodología propuestas por Van-Soest et al. (1991). Cenizas, se determinó de acuerdo a la metodología propuesta por la AOAC (2005). Para determinar la ganancia de peso, se utilizó una balanza digital, las corderas se pesaron cada 15 días.

\section{Análisis estadísticos}

Los datos se analizaron mediante un anova con el procedimiento PROC GLM. La comparación 
Tabla 1. Dietas con diferentes niveles de proteína cruda.

\begin{tabular}{|c|c|c|c|c|c|}
\hline \multirow[b]{2}{*}{ Ingredientes (\%) } & \multicolumn{5}{|c|}{ Tratamiento Proteína cruda (\%) } \\
\hline & 10 & 12 & 14 & 16 & Testigo \\
\hline Maíz grano & 25.00 & 24.00 & 25.00 & 20.00 & 20.00 \\
\hline Pasta de soya & 3.00 & 8.00 & 10.00 & 9.00 & 0.00 \\
\hline Salvado de trigo & 15.10 & 11.30 & 14.10 & 12.00 & 12.00 \\
\hline Pulido de arroz & 15.40 & 13.70 & 13.70 & 3.80 & 0.00 \\
\hline Heno gramíneas MG5 & 26.00 & 25.00 & 20.00 & 20.00 & 36.20 \\
\hline Pasta de coco & 2.00 & 4.00 & 5.00 & 24.00 & 17.00 \\
\hline Melaza & 10.50 & 10.80 & 8.90 & 7.70 & 12.00 \\
\hline Premezcla mineral & 2.00 & 2.00 & 2.00 & 2.00 & 2.00 \\
\hline Sal & 0.50 & 0.50 & 0.50 & 0.50 & 0.50 \\
\hline Carbonato de calcio & 0.30 & 0.30 & 0.30 & 0.30 & 0.20 \\
\hline Urea & 0.20 & 0.40 & 0.50 & 0.50 & 0.10 \\
\hline \multicolumn{6}{|l|}{ Análisis proximal } \\
\hline $\mathrm{PC}$ & 10.06 & 12.18 & 14.09 & 16.04 & 13.39 \\
\hline FDN & 47.18 & 56.47 & 60.72 & 53.96 & 55.47 \\
\hline FDA & 21.61 & 19.74 & 18.07 & 29.51 & 23.09 \\
\hline CENIZAS & 3.67 & 3.79 & 3.63 & 2.85 & 3.56 \\
\hline MS & 92.80 & 92.60 & 92.66 & 92.26 & 84.98 \\
\hline
\end{tabular}

de medias de tratamientos se realizó con la prueba de Tukey (Steel y Torrie 1988).

\section{RESULTADOS}

Durante el periodo del experimento se presentaron condiciones meteorológicas de $561 \mathrm{~mm}$ de precipitación y un promedio de $32.4^{\circ} \mathrm{C}$ máximo y $23.7^{\circ} \mathrm{C}$ mínimo de temperatura (Figura 1). El mes más caluroso fue abril $\left(33.7^{\circ} \mathrm{C}\right)$ y el mes más lluvioso mayo $(283 \mathrm{~mm})$ durante el experimento.

La intensidad de pastoreo afecto la acumulación de MS durante el experimento en el mes de febrero $(p<0.05)$. Independientemente de la intensidad de pastoreo a la que fue sometida la pradera la mayor acumulación de MS y tasa de crecimiento (TC) se obtuvieron en los meses de junio y julio, lo cual se relaciona con la mayor precipitación (Figura 1), que propicio condiciones favorable para que las praderas presentaran mayor cantidad de hojas (Tabla 2). La intensidad de pastoreo afecto significativamente la composición bromatológica, ya que al pastorear el pasto tanzania a una altura residual de 9-11 cm se presentó el mayor contenido de PC, FDN con respecto a la intensidad de $13-15 \mathrm{~cm}$ (Tabla 3).

Dentro de los parámetros productivo hubo diferencias $(p<0.05)$ significativas en el peso final y ganancia diaria de peso, los valores más altos en el peso final se obtuvieron en corderas que pastaron en ambas intensidades y que recibieron alimento con $16 \%$ de PC en la ración, en tanto que las corderas que se alimentaron con el alimento testigo en ambas intensidades de pastoreo los pesos finales fueron similares (Tabla 4). Con respecto a la ganancia de peso los valores mayores se obtuvieron en corderas que pastaron en praderas a una altura residual de 9-13 cm y que se suplementaron con alimento con 14 y $16 \%$ de PC, en cambio las corderas que pastaron en praderas a una intensidad de $13-15 \mathrm{~cm}$ de altura residual la mayor ganancia de peso se obtuvieron en las corderas que se alimentaron con 12 y $14 \%$ de PC.

\section{DISCUSIÓN}

Los valores menores de la acumulación de MS y tasa de crecimiento del pasto Tanzania se obtuvieron en el mes de abril, en este mes no se obtuvieron presencia de tallos, pero si hubo presencia de hoja tal como lo consignan Difante et al. (2011) quienes mencionan que, al cosechar a una intensidad ligera, la pradera presenta buenas reservas de energía y cantidades importantes de hojas residuales, lo que propicia que el índice de crecimiento de nuevas hojas se pueda mantener a nivel alto. La mayor acumulación del 


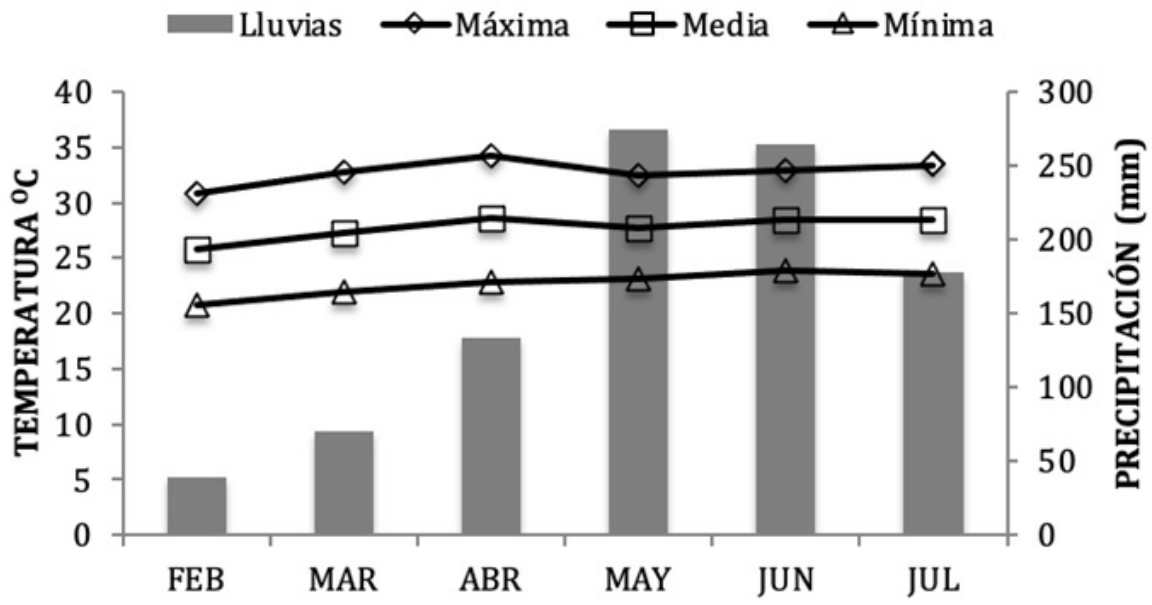

Figura 1. Registro de la precipitación pluvial durante el periodo de muestreo.

Tabla 2. Producción de materia seca, tasa de crecimiento y componentes morfológico del pasto Panicum Maximun cv. Tanzania, manejado a dos intensidades de pastoreo.

\begin{tabular}{|c|c|c|c|c|c|c|c|c|}
\hline \multirow{2}{*}{ Intensidad (CM) } & \multicolumn{8}{|c|}{ Cosechas } \\
\hline & 15 -feb & 08-mar & 29-mar & $19-a b r$ & 10-may & 31-may & 21-jun & 15 -jul \\
\hline \multicolumn{9}{|c|}{ Acumulación de MS (kg MS $\left.{ }^{-1} \mathrm{~h}^{-1}\right)$} \\
\hline $9-11$ & $206.67^{b}$ & $230.00^{a}$ & $226.67^{a}$ & $170.00^{a}$ & $233.33^{a}$ & $260.00^{a}$ & $390.00^{a}$ & $400.00^{a}$ \\
\hline $13-15$ & $316.67^{a}$ & $293.33^{a}$ & $260.00^{a}$ & $173.33^{a}$ & $240.00^{a}$ & $260.00^{a}$ & $503.33^{a}$ & $536.67^{a}$ \\
\hline EEM & 28.21 & 24.27 & 18.19 & 17.01 & 16.26 & 12.90 & 31.58 & 38.76 \\
\hline \multicolumn{9}{|c|}{ Tasa de crecimiento $\left(\mathrm{kg} \mathrm{MS}^{-1} \mathrm{~h}^{-1} \mathrm{~d}^{-1}\right)$} \\
\hline $9-11$ & $9.84^{b}$ & $10.95^{a}$ & $10.79^{a}$ & $8.09^{a}$ & $11.11^{a}$ & $12.38^{a}$ & $18.57^{a}$ & $19.05^{a}$ \\
\hline $13-15$ & $15.08^{a}$ & $13.97^{a}$ & $12.38^{a}$ & $8.25^{a}$ & $11.43^{a}$ & $12.38^{a}$ & $23.97^{a}$ & $25.55^{a}$ \\
\hline EEM & 1.34 & 1.15 & 0.86 & 0.81 & 0.77 & 0.61 & 1.5 & 1.84 \\
\hline \multicolumn{9}{|c|}{ Hojas $\left(\mathrm{kg} \mathrm{MS} \mathrm{h}^{-1}\right)$} \\
\hline $9-11$ & $143.33^{b}$ & $160.00^{a}$ & $226.67^{a}$ & $170.00^{a}$ & $233.33^{a}$ & $260.00^{a}$ & $326.67^{a}$ & $326.33^{a}$ \\
\hline $13-15$ & $226.67^{a}$ & $210.00^{a}$ & $260.00^{a}$ & $173.33^{a}$ & $240.00^{a}$ & $260.00^{a}$ & $360.00^{a}$ & $423.33^{a}$ \\
\hline EEM & 22.02 & 22.91 & 18.19 & 17.01 & 16.26 & 12.90 & 17.82 & 29.86 \\
\hline \multicolumn{9}{|c|}{ 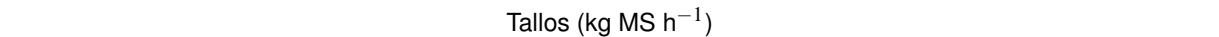 } \\
\hline $9-11$ & $63.33^{a}$ & $70.00^{a}$ & \& & \& & \& & \& & $63.33^{a}$ & $73.33^{a}$ \\
\hline $13-15$ & $90.00^{a}$ & $83.33^{a}$ & \& & \& & \& & \& & $143.33^{a}$ & $113.33^{a}$ \\
\hline EEM & 9.88 & 8.43 & \& & \& & \& & \& & 26.03 & 12.29 \\
\hline \multicolumn{9}{|c|}{ Relación hoja/tallo } \\
\hline $9-11$ & $2.4^{a}$ & $2.6^{a}$ & $\&$ & $\&$ & \& & \& & $5.4^{a}$ & $4.70^{a}$ \\
\hline $13-15$ & $2.6^{a}$ & $2.5^{a}$ & $\&$ & $\&$ & \& & \& & $3.2^{a}$ & $3.84^{a}$ \\
\hline EEM & 0.34 & 0.5 & \& & $\&$ & \& & \& & 0.84 & 0.48 \\
\hline
\end{tabular}

EEM = Error estándar de la media. ${ }^{a b c}=$ Diferente literal minúscula, en cada hilera, indican diferencia $(P<0.05)$. $\&=\operatorname{Sin}$ dato

forraje se le atribuye a las condiciones favorables de precipitación y temperatura que estimularon el crecimiento y rendimiento de las plantas (Festo et al. 2003). Contrariamente en los meses de menor precipitación (Figura 1), las altas temperaturas, fueron las que afectaron el crecimiento del pasto tanzania, dando como resultado una baja producción de forraje (Mattos et al. 2005).
Tabla 3. Composición bromatológica (\%) del pasto Tanzania pastoreado con ovinos Pelibuey.

\begin{tabular}{|c|c|c|c|}
\hline \multirow[b]{2}{*}{ Composición bromatológica } & \multicolumn{2}{|c|}{ Intensidad de pastoreo $(\mathrm{cm})$} & \multirow[b]{2}{*}{ EEM } \\
\hline & $09-11$ & $13-15$ & \\
\hline Proteína & $7.63^{a}$ & $6.64^{b}$ & 0.23 \\
\hline Cenizas & $1.01^{a}$ & $1.01^{a}$ & 0.00 \\
\hline FDA & $58.15^{a}$ & $57.47^{a}$ & 0.20 \\
\hline FDN & $81.62^{a}$ & $77.39^{b}$ & 1.22 \\
\hline MS & $10.36^{b}$ & $11.15^{a}$ & 0.19 \\
\hline
\end{tabular}
cada columna, indican diferencia $(P<0.05)$. 
Tabla 4. Ganancia de peso de corderas Pelibuey alimentados con diferentes niveles de proteína pastando a diferentes intensidades de pastoreo.

\begin{tabular}{|c|c|c|c|c|c|c|c|c|c|c|c|}
\hline \multicolumn{12}{|c|}{ Intensidades de pastoreo } \\
\hline \multirow{2}{*}{ Parámetros } & \multicolumn{5}{|c|}{$09-11 \mathrm{~cm}$} & \multicolumn{5}{|c|}{$13-15 \mathrm{~cm}$} & \multirow[b]{2}{*}{ EEM } \\
\hline & Testigo & 10 & 12 & 14 & 16 & Testigo & 10 & 12 & 14 & 16 & \\
\hline Peso Inicial $(\mathrm{kg})$ & $21.50^{a}$ & $22.75^{a}$ & $19.25^{a}$ & $19.75^{a}$ & $24.25^{a}$ & $23.5^{a}$ & $24.00^{a}$ & $24.25^{a}$ & $24.00^{a}$ & $26.50^{a}$ & 0.7066 \\
\hline Peso Final (kg) & $28.50^{a b}$ & $30.50^{a b}$ & $29.00^{a b}$ & $29.50^{a b}$ & $35.00^{a}$ & $27.00^{b}$ & $30.25^{a b}$ & $28.00^{a b}$ & $31.50^{a b}$ & $34.25^{a b}$ & 0.645 \\
\hline GDP* & $56.25^{a b c}$ & $50.00^{a b c}$ & $62.50^{a b c}$ & $81.25^{a b}$ & $89.58^{a}$ & $48.00^{a}$ & $39.58^{b c}$ & $62.50^{a b c}$ & $87.50^{a}$ & $\mathrm{v} 54.17^{a b c}$ & 4.720 \\
\hline
\end{tabular}

${ }^{\star}$ = Ganancia diaria de peso (gr). EEM = Error estándar de la media. ${ }^{a b c}=$ Diferente literal minúscula, en cada hilera, indican diferencia $(\mathrm{P}<0.05)$. Ración con 10,12, 14, 16\% PC y alimento comercial (Testigo con 16\% PC).

Los valores de PC, FDN y FDA (Tabla 3) se encuentran dentro del rango de referencia para pastos tropicales en México (Améndola 2005, Hare et al. 2013). Por su parte, Verdecia et al. (2008) reportan porcentajes de PC similares a lo obtenido en el presente estudio a edades 60 y 75 días. Silva et al. (2007) al pastorear a $15 \mathrm{~cm}$ de altura residual cada 27 días encontraron hasta $14.45 \%$ de PC. Los resultados encontrado en el presente experimento están cerca del valor mínimo para generar actividad óptima microbiana en el rumen ( $8 \%$ de $\mathrm{PC}$ ) y suministrar aminoácidos al animal (Juárez et al. 2009).

Para los valores de FDN y FDA son similares a los encontrados por Juárez et al. (2009) con respecto a los forrajes Bermuda, Pangola, Guinea y Tanzania. El aumento de FDN y la disminución de PC en forrajes cosechados menos frecuentemente, se debe a que al ser manejado la pradera a intensidades severa y frecuentemente existe remoción de nutrimento estructurales hacia las hojas (Van-Soest 2015); este mismo efecto fue observado en presente trabajo donde se observaron que con el pastoreo selectivo de los ovinos sobre las hojas al inicio del pastoreo, la relación hoja/tallo disminuyó (Tabla 3), debido a que al disminuir las hojas disponibles se expone una parte del tallo, el cual tiene una cantidad de FDN mayor; de acuerdo a Van-Soest (2015) el porcentaje de digestibilidad en el pasto Guinea (Panicum Maximum cv guinea) es de $60-70 \%$ en las hojas y $50-55 \%$ en la parte inferior, de igual modo, la digestibilidad del tallo disminuye desde un 45 al $55 \%$ en la parte más alta y de 35 a $45 \%$ en el punto más bajo.

En México, el peso al sacrificio de los corderos finalizados en sistema intensivo, de razas de pelo es de $35-40 \mathrm{~kg}$ de PV; mientras que para corderos en pastoreo de razas de pelo el rango de peso es de 30 a $40 \mathrm{~kg}$ de PV (Mayren-Mendoza et al. 2018), estos resultados concuerdan con los valores obtenidos en las corderas que pastaron en ambas intensidades y que se alimentaron con 10, 14 y $16 \%$ de PC en la ración. El manejo a diferentes intensidades de pastoreo afectó significativamente la ganancia diaria de peso (GDP). Los valores mayores se obtuvieron en las corderas que se alimentaron con 14 y $16 \%$ PC en la ración y que pastaron en ambas intensidades (Tabla 4), estos resultados son mayores a los valores mínimo reportado para el trópico en pastoreo extensivo ( $50 \mathrm{~g} \mathrm{Animal}^{-1}$ día $^{-1}$ ) la cual depende de la disponibilidad y calidad del forraje (Sosa et al. 2004). Por su parte Hinojosa et al. (2013) reportan ganancia diaria de peso de $116 \mathrm{~g}_{\text {animal }}{ }^{-1} \mathrm{día}^{-1}$ en machos alimentados con caña de azúcar (Saccharum officinale) y alimento comercial al $15 \%$ de PC en sistema estabulado con 190 días de engorda en cruzas de $\mathrm{F} 1$ de pelibuey $\mathrm{x}$ black belly. Por su parte Macías et al. (2010) obtuvieron ganancias de 206 $\mathrm{g}$ animal ${ }^{-1}$ día $^{-1}$ con un porcentaje de proteína de iniciación de $19.6 \%$ y de finalización de $16.4 \%$ con 100 días en corderos machos y hembras de pelibuey puros, pelibuey $\mathrm{x}$ katadhin $\mathrm{y}$ pelibuey $\mathrm{x}$ dorper. En los trabajos mencionados, se observa que la cantidad de proteína ofrecida en la dieta es un factor importante para ganancia de peso, variando entre hembras y machos, y entre razas y sus cruzas con valor mínimo de $16 \%$ de PC en la ración según lo establecido por la NRC (2007). Lo cual, concuerda con el valor encontrado en los tratamiento de $16 \%$ de proteína y con altura residual de $9-11 \mathrm{~cm}\left(89.58 \mathrm{~g}_{\text {animal }}{ }^{-1} \mathrm{día}^{-1}\right)$. 


\section{CONCLUSIONES}

El pasto Tanzania presentó buena producción de materia seca y componentes morfológicos al ser pastoreada a una intensidad de defoliación $13-15 \mathrm{~cm}$. Sin embargo, los valores nutritivos mayores se obtuvieron en las praderas que se pastorearon a una in- tensidad de 9-13 cm de altura. Las corderas que se alimentaron con $16 \%$ PC en la ración presentaron las mayores de ganancia diaria de peso. Los estudios de engorda de ovinos en pastoreo son escasos, por lo cual es necesario continuar trabajando en esta línea de investigación con diversas especies forrajeras.

\section{LITERATURA CITADA}

Améndola MR, Castillo E, A Martínez P (2005) Perfiles por país del recurso pastura/forraje. FAO. Roma, Italia. $58 \mathrm{p}$.

AOAC (2005) Official Methods of Analysis. Edition 18. Association of Official Analytical Chemists. Washington, 29 DC, EE. UU. 1574p.

Avendaño L, Álvarez FD, Salomé J, Correa A, Molina L, Cisneros FJ (2004) Evaluación de algunos rasgos productivos del borrego Pelibuey en el noroeste de México. Resultados preliminares. Revista Cubana de Ciencia Agrícola 38: 131-136.

Cruz-Sánchez OE, Cruz-Hernández A, Gómez-Vázquez A, Chay-Canul AJ, Joaquín-Cansino S, De la CruzLázaro E, Márquez-Quiroz C, Osorio-Osorio R, Hernández-Garay A (2018) Producción de forraje y valor nutritivo del pasto Mulato II (Bracharia hibrido 36087) a diferentes régimen de pastoreo. Revista Agroproductividad 11: 18-23

Difante GS, Júnior DN, Da Silva SC, Euclides VPB, Montagner DB, Silveira MCT, Pena KD (2011) Características morfogênicas e estruturais do capim-marandu submetido a combinações de alturas e intervalos de corte. Revista Brasileira de Zootecnia 40: 955-963.

Difante GS, Euclides VPB, Nascimento Jr D, Da Silva SC, Barbosa RA, Torres jr RAA (2010) Desempenho e conversão alimentar de novilhos de corte em capim tanzânia submetido a duas intensidades de pastejo sob lotação rotativa. Revista Brásileira de Zootecnia 39: 33- 41.

Festo JM, Sabed NA, Jeremy AR (2003) The impact of temperature on leaf appearance in bamba groundnut landraces. Journal Crop Science 43: 1375-1379.

García E (2004) Modificaciones al Sistema de Clasificación Climática de Koppen. Quinta Edición. Instituto de Geografía. UNAM. México. 69p.

Hare MD, Phengphet S, Songsiri T, Sutin N, Stern E (2013) Effect of cutting interval on yield and quality of two Panicum maximum cultivars in Thailand. Tropical Grasslands 1: 87-89.

Hernández GA, Martínez HP, Mena UM, Pérez PJ, Enriquez QJ (2002). Dinámica del rebrote en pasto insurgente (Brachiaria brizantha Hochst. stapf.) pastoreado a diferente asignación en la estación de lluvia. Técnica Pecuaria México 40: 193-205.

Hernández CL, Ramírez EB, Guerrero IL, Hernández OM, Crosby MG, Hernández M C (2009) Effects of crossbreeding on carcass and meat quality of Mexican lambs. Arquivo Brasileiro de Medicina Veterinária e Zootecnia 61: 475-483.

Hinojosa CJA, Oliva HJ, Torres HG, Segura CJC (2013) Comportamiento productivo de corderos F1 Pelibuey $x$ Blackbelly y cruces con Dorper y Katahdin en un sistema de producción del trópico húmedo de Tabasco, México. Archivos de Medicina Veterinaria 45: 135-143. 
Inyang U, Vendramini MB, Sollenberger BL, Adesogan LA, Lupha A (2010) Forage species and stocking rate effects on animal performance and herbage responses of 'Mulato' and Brachiaria grass pasture. Journal Crop Science 50: 1079-1085.

Juárez RAS, Cerillo SMA, Gutiérrez OE, Romero TEM, Colín NJ, Bernal BH (2009) Estimación del valor nutricional de pastos tropicales a partir de análisis convencionales y de la producción de gas in vitro. Técnica Pecuaria México 47: 55-67.

Macías CU, Álvarez VFD, Rodríguez GJ, Correa CA, Torrentera ONG, Molina RL, Avendaño RL (2010) Crecimiento y características de canal en corderos Pelibuey puros y Cruzados F1 con razas Dorper y Katahdin en confinamiento. Archivos de Medicina Veterinaria 42: 147-154.

Mattos SJ, Gomide JA, Martinez CA (2005) Crescimento de espécies de Brachiaria sob déficit hídrico e alagamento a campo. Revista Brasileira de Zootecnia 34: 755-764.

Mayren-Mendoza FJ, Rojas-García AR, Maldonado-Peralta MA, Ramírez-Reynoso O, Herrera-Pérez J, TorresSalado N, Sánchez-Santillán P, Bottini-Luzardo MB, Hernández-Garay A (2018) Comportamiento productivo de ovinos Pelibuey en pastoreo suplementados con follaje de Guazuma ulmifolia Lam. Agroproductividad 11: 29-33.

Moreno GMB, Buzzulini C, Borba H, Costa AJ, Lima TMA, Dourado JFB (2011) Efeito do genótipo e do teor de proteína da dieta sobre a qualidade da carne de cordeiros. Revista Brasileira de Saúde e Produção Animal 12: $630-640$.

Nantes NN, Euclides PBV, Montagner BD, Lempp B, Barbosa AR, Gois OP (2013) Desempenho animal e características de pastos de capim-piatã submetidos a diferentes intensidades de pastejo. Pesquisa Agropecuária Brasileira 48: 114-121.

NRC (2007) Nutrient Requirements of Small Ruminants. Sheep, goats, cervids, and new world camelids. Nutrient Requirements Council. Editión The National Academy Press. Washington, D.C., USA. 362p.

Palma LD, Cisneros DJ (1996) Plan de uso sustentable de los suelos de Tabasco. Fundación Produce Tabasco. Villahermosa Tabasco, México. 55p.

SIAP (2014) Resumen nacional de la producción pecuaria. Secretaria de Información Agroalimentaria y Pesquera. http://infosiap.siap.gob.mx/repoAvance_siap_gb/pecResumen.jsp. Fecha de consulta 3 de octubre de 2014.

Silva MDC, dos Santos MVF, de Mello ACL, Lira MDA, Ferreira RLC, Moreira JN, de Freitas EV, Farias I (2007) Aspectos produtivos e composição química dos capins Tanzânia e Mombaça submetidos a diferentes manejos. Pesquisa agropecuaria pemambuco 14: 11-18.

Sosa REE, Pérez RD, Ortega, RL, Zapata BG (2004) Evaluación del potencial forrajero para la alimentación de ovinos. Técnica Pecuaria en México 42: 129- 144.

Steel RG, Torrie JH (1988) Bioestadística. Principios y procedimientos. 2da. edit. McGraw Hill. México. 622p.

Ulloa AR, Gayosso VA, Alonso MRA (2009) Origen genético del ovino criollo mexicano (Ovis aries) por el análisis del gen del Citocromo C Oxidasa subunidad I. Técnica Pecuaria México 47: 323-328.

Van-Soest PJ (2015) Evaluación de forrajes y calidad de los alimentos para rumiantes. http://tiesmexico.cals. cornell.edu/courses/shortcourse2/minisite/pdf/Calidad de Alimentos para Rumiantes/articulo Van Soest.pdf. Fecha de consulta 23 de septiembre de 2015.

Van-Soest PJ, Robertson JB, Lewis BA (1991) Methods for dietary fiber, neutral detergent fiber, and nonstarch polysacharides in relation to animal nutrition. Journal Dairy Science 74: 3583-3597. 
Verdecia DM, Ramírez LJ, Leonard I, Pascual Y, López Y (2008) Rendimiento y componentes del valor nutritivo del Panicum maximum cv. Tanzania. Revista electrónica de veterinaria 9: 1-9

Zanine ADM, Nascimento Júnior DD, Sousa BMDL, Silveira MCTD, Silva WLD, Santos MER (2013) Tillering dynamics in Guinea grass pastures subjected to management strategies under rotational grazing. Revista Brasileira de Zootecnia 42: 155-161. 